\title{
Los Grandes Olvidados: Las Personas Mayores en el Entorno Rural
}

\section{The Great Forgotten Issue: Older People in Rural Context}

\author{
Pilar Monreal Bosch, Arantza del Valle Gómez y Bernat Serda Ferrer \\ Universidad de Girona
}

\begin{abstract}
Resumen. La heterogeneidad en las personas mayores es enormemente variada, pudiéndose distinguir perfiles en función del sexo, la edad, la situación económica, los niveles culturales, la forma de hábitat, la composición de la estructura familiar, el estado de salud, la forma de envejecer, etc. Los objetivos de este artículo son identificar formas de vida de envejecer en el entorno rural y concretar propuestas de intervención que conduzcan a favorecer estilos de vida más activos y participativos. En total se hicieron 7 entrevistas en profundidad a expertos, 21 entrevistas a personas mayores, 5 entrevistas a profesionales y dos grupos focales de profesionales y agentes sociales significativos. En los resultados se identifican 4 estilos diferenciados de envejecimiento como una oportunidad para el cambio, una etapa natural de la vida, un momento a compensar y un momento de abandono. Para concluir concretamos propuestas de intervención que conduzcan a favorecer estilos de vida más activos y participativos en los diferentes contextos y que sirvan de ayuda a los profesionales responsables de los servicios de atención a las personas mayores.
\end{abstract}

Palabras clave: entorno rural, estilos de vida, envejecimiento activo.

\begin{abstract}
Ageing is a heterogeneous subject being been able to distinguish profiles in function of sex, age, economic situation, cultural levels, habitat, family structure, health, etc. The objectives of this article are to identify forms of life to age in the rural environment and to concretize intervention proposals to increase active and participatory ways of life. 7 indepth interviews to experts, 21 to older people, 5 to professionals and two focal groups of professionals and significant social agents were performed to collect data. Results identify 4 styles of aging: an opportunity for the change; a natural phase of the life; a moment to compensate and; a moment of abandonment. Conclusions summarize intervention proposals to increase active and participatory ways of ageing in different contexts to help professionals responsible for the services of older people care.
\end{abstract}

Key words: rural context, life styles, active aging.

\section{Introducción}

Las personas mayores al igual que los jóvenes tienen capacidades diferentes para enfrentarse a la

La correspondencia sobre este artículo puede dirigirse a la primera autora a la Universidad de Gerona. Departamento de Psicología. Emili Grahit, 77. 17071 Gerona, o a través del correo electrónico: pilar.monreal@udg.edu vida y aceptar sus situaciones personales; así coincidimos con Neugarten (1999), cuando afirma que envejecemos de la misma manera que hemos vivido. La persona mayor que envejece de forma óptima es aquella que se mantiene activa y consigue resistir al empequeñecimiento de su mundo social, conserva las actividades que realizaba en su vida adulta tanto tiempo como le es posible, encontran- 
do sustitutos para aquellas que se ve forzada a abandonar.

La Organización Mundial de la Salud, en 2002, ya acordó el concepto de "envejecimiento activo" como el proceso de optimización de las oportunidades de salud, participación y seguridad con el fin de mejorar la calidad de vida a medida que las personas envejecen. El término activo hace referencia a una participación continua en las cuestiones sociales, económicas, culturales, espirituales y cívicas, no sólo a la capacidad para estar físicamente activo o participar en la mano de obra, y el término salud se refiere al bienestar físico, mental y social expresado por la OMS en su definición de salud.

No existe un único patrón de envejecer con éxito, diferentes formas de envejecer se pueden considerar como una adaptación satisfactoria. Las personas envejecen, no solamente de formas diferentes, sino que la gama de diferencias se hace más amplia a medida que pasa el tiempo. Además el curso de la vida humana $y$, en consecuencia, de los patrones de envejecimiento es diferente en las distintas sociedades, en los distintos grupos y en diferentes épocas de la historia. Los patrones de la edad adulta tardía (vejez) se ven afectados aunque no determinados por la experiencia anterior, en consecuencia, el estudio del envejecimiento debe verse como una interrelación constante del tiempo vital, el tiempo definido socialmente y el tiempo histórico. (Baltes, 1998).

De acuerdo con el construccionismo social, las personas damos una importancia extraordinaria a nuestras interacciones cotidianas y las consideramos activamente responsables de la producción de las formas de conocimiento, que a menudo damos por descontadas, y de los fenómenos sociales correspondientes (Gergen, 1985).

En lugar de pensar que el individuo y la sociedad son los dos elementos opuestos de una dicotomía, habríamos de considerarlos componentes inseparables de un sistema, ya que ninguno de los dos tendría sentido sin el otro. Así, el sistema individuo/ sociedad es una nueva unidad de estudio en que ninguno de los dos términos no hace referencia a nada que pueda ser entendido aisladamente. Se entiende como un sistema "ecosistema" que comprende tanto el organismo como su entorno, tanto el individuo como la sociedad, o sea ver la relación del individuo y la sociedad como un ecosistema.

Lo más próximo es la zona de vida cotidiana directamente accesible a su manipulación corporal. Esa zona contiene el mundo que está a su alcance, el mundo en el que actúa a fin de modificar su realidad, el complejo de interrelaciones dentro del entorno inmediato, y es la zona con la que las personas se sienten más profundamente interesadas. Esta zona coincide con el concepto de microsistema de Brofenbrenner (1987), por ejemplo, el mundo de la estación de servicio, para un mecánico.

En un segundo nivel, la realidad de la vida cotidiana de las personas se construye también, aunque menos directamente, a través de lo que ocurre en contextos que sin su participación directa, tienen la capacidad de influir en su vida cotidiana. Es decir, los laboratorios de prueba de la industria automovilística de Detroit, puede afectar eventualmente la vida cotidiana de un mecánico, aunque este no participe jamás en las actividades que allí se desarrollan. Esta zona coincide con el concepto de exosistema de Brofenbrenner (1987).

Por último, nuestro pensamiento responde a conceptos colectivos más amplios. Las ideas, los valores, y las creencias de la sociedad en el seno de la cual hemos nacido configuran las imágenes que poblaran nuestro pensamiento y determinan nuestra percepción dialéctica del mundo, las maneras de representarnos y de representar nuestro entorno social. Configuran no sólo aquello que pensamos y decimos, sino también nuestros sentimientos, nuestros deseos y nuestros actos. Este tercer nivel de la vida cotidiana coincide con el concepto de macrosistema propuesto por Brofenbrenner (1987).

La realidad cotidiana del mundo de la vida incluye no solo la "naturaleza" experimentada, sino también el mundo social (y por ende el mundo cultural) en el cual se encuentra la persona, el mundo de la vida no se crea a partir de los objetos y sucesos simplemente materiales que las personas hallan en su entorno. Ahora bien, aunque las personas reconocen que la realidad de la vida cotidiana incluye zonas que no le son accesibles directamente, hay que destacar que su mundo de la vida cotidiana esta principalmente determinado por lo que hace, lo que ya ha hecho o lo que piensa hacer en él. 
Diversos estudios (Horgas, Wilms y Baltes, 1998; Fidler, 1996) nos muestran como los estilos de vida se configuran a partir de la confluencia de influencias socio-culturales, biológicas y personales y es, por lo tanto, necesario considerar los estilos de vida como parte de una dimensión colectiva y social (Bibeau y col 1985). Desde esta perspectiva integral, los estilos de vida no pueden ser aislados del contexto social, económico, político y cultural al cual pertenecen, y deben ser acordes a los objetivos del proceso de desarrollo de alcanzar y/o mantener el bienestar de la persona humana en la sociedad a través de la satisfacción de sus necesidades de expresión, creatividad, participación, igualdad de condiciones de convivencia, y autodeterminación, entre otras (Smits et al., 1999; Whalley y Deary, 2001). Los estilos de vida se definen como un conjunto de comportamientos y/o actitudes que configuran la manera en que vive una persona. Es decir, reflejan las actitudes, los valores o la visión del mundo de un individuo (Fidler, 1996). Esta configuración de la vida cotidiana tiene, por un lado, una gran resistencia al cambio y por otro, una gran importancia predictiva en el desarrollo durante la vejez. De ahí la relevancia y el interés de su estudio para los profesionales de servicios orientados a las personas mayores activas.

Nuestra cambiante sociedad ha traído consigo modificaciones en los significados sociales de la edad, los limites entre los distintos periodos de la vida han perdido nitidez, han aparecido nuevas definiciones de los grupos de edad, nuevos patrones en las cronologías de los principales acontecimientos de la vida y nuevas inconsistencias en lo que se considera un comportamiento adecuado a cada edad.

Curiosamente se reconoce en las personas mayores el valor de la experiencia, pero no se considera que en la vida cotidiana una de las fortalezas de esa experiencia se traduce en términos de capacidad de adaptación, de manejarse en situaciones diversas, de afrontar retos variados y en definitiva de aprender.

El estudio del proceso de envejecimiento en entornos rurales se presenta hoy como uno de los retos más importantes (Golant, 2004). El envejecimiento en entornos rurales se caracteriza por afectar a toda la comunidad, tener una población de personas mayores mucho más elevada que en el ámbito urbano, presentar una menor proporción de personas que viven solas y una mayor dificultad de acceso a cualquier servicio (Hink, 2004). Además, los mayores que viven en un entorno rural, sienten como situación más temida la soledad y desconocen en gran medida la existencia de servicios, como pueden ser los centros de día o el servicio de estancias temporales en residencias. Por otro lado, la satisfacción que sienten por sus relaciones sociales es mucho mayor que la de las personas mayores que viven en grandes ciudades. (Departament de Benestar Social, 2002).

Estos rasgos definitorios del entorno rural, nos plantean la necesidad de conocer con mayor profundidad los patrones de envejecimiento de las personas mayores en entornos rurales y urbanos desde su perspectiva, así como la de sus familias, para identificar aquellas acciones que se consideran pertinentes para promover la autonomía personal, prevenir la dependencia y potenciar un envejecimiento exitoso teniendo en cuenta las diferencias entre los entornos.

Coincidimos con diversos autores (García Sanz, 1996; Monreal y Vilà, 2008) en definir el espacio rural como un área en la que existe un modo particular de utilización del espacio y de la vida social, caracterizada por:

1. Una densidad relativamente débil de habitantes y de construcciones, lo que determina un predominio de los paisajes vegetales.

2. Un uso económico del suelo de predominio agro-pastoril.

3. Un modo de vida de sus habitantes marcado por su pertenencia a colectividades de tamaño limitado, en las que existe un estrecho conocimiento personal y fuertes lazos sociales.

4. Una especial relación que los habitantes mantienen con el espacio, favoreciendo un entendimiento directo y vivencial del medio ecológico.

5. Una identidad campesina.

Por lo tanto, como forma de hábitat se identifica lo rural con núcleos de población pequeños, núcleos de población en los que la gente se conoce, se llama por su nombre o su apodo (relaciones cara a cara). En la sociedad rural, las personas se relacionan de otra manera, en esta sociedad funcionan de forma 
muy marcada los roles y el status de adscripción, por encima de los logros personales; normas de convivencia que poco a poco se han ido imponiendo. El concepto de tiempo y del espacio, son diferentes. No existe la idea de un horario fijo, ni la división entre tiempo de ocio y tiempo de negocio. Se trabaja pero en cualquier momento se puede tomar un descanso.

García Sanz (1997), dice que los mayores rurales no son solamente una parte proporcionalmente más numerosa que la de los urbanos, sino que en el medio rural se envejece de forma diferente porque el contexto en el que se desarrolla este hecho es muy distinto. Todo ello obliga a buscar soluciones que deben encontrarse integradas y adaptadas a este medio.

La heterogeneidad en las personas mayores es enormemente variada, pudiéndose distinguir perfiles en función del sexo, la edad, la situación económica, los niveles culturales, la forma de hábitat, la composición de la estructura familiar, el estado de salud, la forma de envejecer, etc. En este estudio queremos fijarnos en las diferencias según el hábitat, el envejecimiento rural y sus notas características.

Pero si se quiere profundizar en los problemas de los mayores no es suficiente conocer cuántos son, sino quiénes son. En este sentido hay tres notas que es importante reseñar: 1) los mayores rurales están más envejecidos, tienen una esperanza de vida mayor; (IMSERSO 2009); 2) los mayores rurales están relativamente más masculinizados, en el mundo rural abundan mucho más las mujeres que los hombres, pero las diferencias no son tan abultadas como en el mundo urbano, García Sanz (1997). A medida que se van cumpliendo años, tanto la población mayor rural como la urbana se feminizan, pero con la salvedad de que la población urbana se feminiza mucho más que la rural, y 3) la soledad se puede subsanar mucho mejor en el medio rural con la ayuda vecinal que en el urbano, en donde se ha roto este tipo de relaciones, o no funciona con la misma regularidad (Rodríguez, 1995).

Los mayores rurales no se han caracterizado precisamente por la movilidad, por eso suelen oponerse a cambiar de lugar, aunque sea para dar respuesta adecuada a su dependencia. Mucha más aceptación tiene el formar familias extensas en las que conviven varias generaciones; esta forma de familia es todavía importante en algunas regiones, como Cantabria, Cataluña y Galicia (la pervivencia de estas formas familiares están relacionadas con los sistemas de herencia que transmiten toda la propiedad a un solo heredero, que, en recompensa, asume el cuidado de los ancianos).

Los objetivos que nos planteamos son:

1. Identificar formas de vida de envejecer en el entorno rural.

2. Concretar propuestas de intervención que conduzcan a favorecer estilos de vida más activos y participativos en los diferentes contextos y que sirvan de ayuda a los profesionales responsables de los servicios de atención a las personas mayores.

\section{Procedimiento}

La recogida de datos se ha realizado a través de grupos focales y entrevistas individuales en profundidad. Estos procedimientos se han utilizado para generar información contando con la perspectiva de las personas del estudio. Y constituyen una fuente de significados imprescindible ya que, de esta manera, se podrán interpretar aspectos de la cuestión a estudiar que no son directamente observables pero si definibles. Es así como se garantiza una visión global de los puntos de vista de las personas del estudio. (Taylor y Bodgan, 1986).

En total se hicieron 7 entrevistas en profundidad a expertos, 21 entrevistas a personas mayores, 5 entrevistas a profesionales y dos grupos focales de profesionales y agentes sociales significativos. Con un total de 53 participantes.

Se realizó un muestreo intensivo por representatividad teórica de máxima variación (Morse, 1994) teniendo en cuenta los criterios muestrales de inclusión para los diferentes tipos de informantes. Para la realización del estudio se seleccionó la comarca de l'Alt Empordà (Girona), por sus características: es una comarca principalmente rural, con poblaciones costeras, de interior, de frontera y con un núcleo urbano importante, con una población envejecida.

El procedimiento para el análisis de los datos fue el Método de Comparaciones Constantes y la 
Triangulación de los resultados generados por las diferentes técnicas e informantes.

\section{Resultados}

El estilo de vida de una persona implica y refleja sus metas, valores y preferencias, a través de las actividades que elige realizar, hasta cierto punto, intencionalmente (Brandtstadter y Lerner, 1999).

En el material analizado podemos identificar cuatro estilos de vida en la vejez:

\section{a) La vejez como una oportunidad para cambio}

La vejez se contempla aquí como una oportunidad de tener tiempo para hacer cosas que son vistas como deseables. Las personas mayores que se incluyen en este perfil ven la vejez como una liberación, una etapa en la que las obligaciones son secundarias y lo principal es aprovechar la oportunidad para crear o desarrollar un proyecto vital largamente aplazado. Este perfil esta formado por personas que se sienten con capacidad para realizar actividades de todo tipo: de placer, familiares, personales, sociales etc., que pueden y quieren disfrutar de sus aficiones, sus relaciones sociales y familiares y, que tienen objetivos vitales que quieren conseguir antes de morir. Sienten que por fin disponen de tiempo para realizar aquellas actividades que no pudieron hacer antes por falta de tiempo. El contexto inmediato aparece aquí como una fuente de actividades significativas para mantener una participación que refuerza su identidad personal y social y lo aprovechan organizando su vida cotidiana entorno a sus propias aficiones y a su desarrollo personal. La muerte también está presente pero como el punto final antes del cual todavía se pueden conseguir objetivos vitales que se consideran importantes y que no se había tenido tiempo de desarrollar. Se quiere disfrutar del tiempo que queda.

Los viejos jóvenes se están convirtiendo en una parte integrante de la vida cotidiana y no tienen una edad en particular sino unas características sociales asociadas a la participación y unas características de salud asociadas a la autonomía.
Los viejos jóvenes es un grupo que se esta incrementando con rapidez formado por jubilados y sus cónyuges, que están en plenas facultades mentales y físicas y cuyo principal rasgo distintivo es tener un nuevo tiempo de ocio. Estas personas están relativamente sanas, tienen una situación económica relativamente acomodada, cada vez cuentan con una mejor formación y son activos socialmente e importantes consumidores de bienes y servicios. Son personas que buscan formas interesantes de utilizar su tiempo, tanto para obtener autosatisfacción como para contribuir al bienestar de sus comunidades, y actualmente representan un potencial desaprovechado en nuestra sociedad.

"Se tiene que preparar unos años antes, pensar qué hará, una cosa que nunca has podido hacer porque trabajas y ahora puedes dedicarte." (24munu, 64:64).

"Es un colectivo que cuando nos retiramos a 65 años ahora, las cosas han evolucionado y estamos capacitados aún para ayudar a la sociedad, una sociedad que a mi me da la sensación que la necesita mucho esta ayuda" (4icPG, 18:18).

Este estilo de envejecimiento en el entorno rural, por un lado, en la zona de interior está protagonizado principalmente por antiguos emigrantes rurales que, una vez jubilados deciden regresar a su lugar de origen. Este tipo de migración se produce cuando las personas mantienen recursos sociales (familiares, amigos) o materiales (propiedades) en el pueblo, recursos que han podido mantener a través de su tiempo de vacaciones. Contribuyen a la recuperación de los pueblos aportando otras maneras de envejecer, y ayudando a construir un sentido de continuidad entre el pasado, el presente, y el futuro, de especial relevancia para las personas mayores.

“Al no haber vida marchamos. ¿Que pasa? Si tienes la casa de los padres o si te has ganado la vida y las cosas te han ido un poco bien pues ee la casa, has tenido una casa vieja y poco a poco pues la has ido arreglando." (10h67i, 49:49).

Por otro lado es un estilo de envejecimiento más común en las zonas costeras donde, aunque no se da a través del retorno, la llegada de un numero elevado de personas vinculadas a los servicios y al ocio 
ha favorecido la colonización de valores urbanos, la incorporación de nuevas formas de vida y la introducción de un nuevo modelo de envejecimiento actualizado que lo diferencia de las generaciones anteriores.

"Que antes las personas, yo me acuerdo de mi suegra, que se pasaba el dia sentada en la silla y tenia mi edad y era muy vieja, iyo la veía muy vieja!" (1d81c).

\section{b) la vejez como una etapa "natural" de la vida}

La vejez es vista desde este perfil como una etapa más de la vida, en la que la continuidad con el resto de las etapas vividas es lo que le da sentido. La vejez forma parte del curso "natural", esperado y, por lo tanto, no problemático, de la vida. Para estas personas durante la vejez se mantienen y desarrollan los valores y las creencias que han orientado la vida adulta. Considerando que cambiarán las actividades, que pasarán a ser las "normales" de las personas mayores, pero que los objetivos vitales que han guiado las etapas anteriores se pueden continuar desarrollando. La vejez se considera, pues, un momento más de la vida, equivalente a otros, que no es en sí mismo ni negativo ni positivo, y al cual se hará frente con las mismas estrategias que hasta el momento. La continuidad se manifiesta, también, en las actividades que tendrán relación con las que se hayan hecho antes, y que son las consideradas como propias para las personas mayores, valoradas como significativas y gratificantes, lo cual implica una predisposición positiva al proceso de envejecimiento y de dejarse llevar por lo típico lo natural alejándose de los perfiles caracterizados por la iniciativa propia (la vejez como oportunidad para el cambio, la vejez como un momento a compensar)

Dos procesos caracterizan esta posición: la aceptación y el ajuste. La aceptación caracterizada por la percepción de la vejez como algo inevitable, esperado, propio del vivir, no se siente dolor y se mantiene una actitud positiva delante del avance de la vida y del paso del tiempo. El ajuste, implica la articulación, aunque pasiva, de una vida cotidiana que se amolda a una visión típica y socialmente prevista de las actividades, funciones y responsabilidades de las personas mayores. Este ajuste se realiza de forma "natural" dentro del entorno familiar, así en este perfil encontramos personas que aunque pueden tener niveles de actividad altos (llevar-traer nietos al colegio, cuidar nietos, etc.) corren el riesgo de quedar aislados de personas de su generación y de reducir su red de relaciones sociales vinculadas a la amistad.

"Hay que adaptarse, nadie te dará nada...te ayudaran, algunos te ayudaran pero nadie te dará nada, eh? A ver, mi familia, yo sé que no me faltará nunca nada, eh? Por la parte, dijéramos de mi mujer, las relaciones son perfectas, inmejorables, eh? no, porque a ver, no soy conflictivo, me adapto, más bien me adapto a la vida de ellos que a la mía. "(4h73c, 279:279).

Esta forma de vida en el envejecimiento rural, se organiza entorno a los roles de género más tradicionales. Los hombres habitan la esfera pública de la vida, el mundo del trabajo y de la competencia, mientras que la vida cotidiana de las mujeres, en cambio, se suele centrar en la familia y forma parte de una red de relaciones interconectadas que no se basan en la competencia sino en la cooperación y el afecto mutuo. Las mujeres frecuentan más "otros hogares" y los espacios públicos como los mercados, iglesias y suelen utilizar como lugares colectivos los paseos para caminar, los domicilios particulares, mientras que los hombres se reúnen en lugares socialmente construidos como puntos de encuentro y mencionan más bares y restaurantes y huerto y granjas.

Ella está aquí arriba, jugando a las cartas con unas compañeras y yo estoy abajo (bar), también jugando, a echar la partida y ya me quedo abajo un ratito o me subo aquí arriba y ya nos vamos para la casa. (3h73c., 80:80).

En esta forma de vida, las personas mayores llevan a cabo actividades consideradas típicas de la persona mayor, entre ellas, especialmente el cuidado de los nietos. En las zonas de costa y en los municipios más grandes, donde la vida de las familias jóvenes es claramente "moderna" y "líquida" encontramos que las personas mayores dedican una parte importante del tiempo al cuidado de los nietos con la finalidad de ayudar económicamente a sus hijos "así no pagan a nadie". Estos abuelos sustituyen temporal o regularmente a los padres, situación que supone un condicionante o una ruptura en la vida cotidiana de la persona 
mayor que realiza una actividad exclusivamente de acompañamiento del nieto pero no una actividad propia "voy al parque porque tengo al nieto".

Por otro lado en las zona de interior, esta relación típica abuelos-nietos se construye con la finalidad de mostrar a los nietos de hacerles vivir y disfrutar la cultura, los valores y el estilo de "la familia" y por lo tanto, velar por la continuidad de la historia familiar y de la comunidad acercándoles a una realidad social construida a partir de las "relaciones cara a cara" significativas para ambos.

\section{c) la vejez como un momento a compensar}

Esta posición se caracteriza por su actitud compensatoria delante de las dificultades y los retos que produce la vejez, principalmente la soledad. Compensatoria porque no se anticipan ni se previenen los retos antes de que aparezca un problema asociado (por ejemplo el empequeñecimiento de la red social, la soledad). Pero una vez aparece el problema se ponen en marcha mecanismos para solventarlo (por ejemplo ir al casal). Se esfuerzan en modificar lo menos posible sus rutinas y relaciones sociales, sólo cambian aquello que es necesario. Tienen dificultades para asumir cambios significativos. La vejez desde esta posición se entiende como un momento vital caracterizado por la gran cantidad de problemas a los que hacer frente. Este perfil aunque comparte una visión negativa de la vejez con el perfil llamado de momento de abandono, la afronta de forma activa, con iniciativa propia para solucionar los problemas, pero no motivados por un proyecto vital aplazado como en el primero de los perfiles (oportunidad para el cambio) sino por la necesidad de afrontar sus problemas en la vida cotidiana.

"Cuando veo a las amigas nuestras, que ahora no tienen a su marido por desgracia,... y...muchos días que tienen de esto, nosotros las acompañamos a su casa ... y la dejamos...y yo cuando baja y cierra la puerta le digo a Lluís: "Y es que es horrible, ¿eh?” Toda sola allí... si ella pobrecita, ¿sabes qué hace? Esa si se apunta a todo, a las excursiones... a todo... porque lo que no quiere es estar en su casa... Estar sola. Y sino, cuando llega a casa, al sofá y un vaso de leche y una pastilla, se duerme y no se entera de nada, por desgracia..." (8d65u, 243:245).

En este estilo de vida la participación en espacios sociales organizados tienen mucha presencia en la vida cotidiana de las personas mayores sobre todo de las personas que viven solas, que ven en ellos un espacio para las relaciones, el entretenimiento y las oportunidades; aunque son las mujeres quienes más aprovechan estas oportunidades. Por otro lado los hombres tienden a participar más de espacios naturales de encuentro, vinculados habitualmente a su actividad productiva, por ejemplo el puerto en las zonas de costa o el huerto y el campo en las zonas de interior.

\section{d) la vejez como un momento de abandono}

La vejez se considera desde esta perspectiva como un acontecimiento inevitable en la trayectoria vital. La vejez se define como una pérdida dolorosa, una etapa sin reconocimiento social donde no se es útil ni/o válido, una etapa sin objetivos, la etapa antes de la muerte. Una actitud de abandono y resignación caracteriza esta posición, ya que existe una despreocupación por el contexto inmediato que les lleva al empequeñecimiento total de su vida cotidiana. Su vida cotidiana se caracteriza por el desarrollo prácticamente en exclusiva de actividades necesarias para la supervivencia. Estas actividades se consideran poco gratificantes, pero tampoco se construyen/buscan alternativas significativas. Las personas mayores en este perfil tienen un conocimiento pobre de las opciones disponibles en su entorno. La muerte esta presente como el final seguro de esta etapa que se define como "antesala de la muerte" lo que les lleva a aspirar a la supervivencia desde una posición resignada, pasiva que se observa en el abandono del cuidado físico, de las relaciones sociales e incluso de la vida familiar.

"Pero que yo... yo lo que tengo no se lo debo a nadie, he trabajado mucho, muchísimo, he llorado mucho, he pasado muchas penas... $Y$ ahora que podría estar un poquito mejor, pues ya está todo viejo... y ya está. No tengo otra cosa, hija. ¿Qué vas a protestar?... Y una 
mujer ya mayor... cuando es una persona joven se puede defender, pero una mujer mayor, que te dan un empujón y te vas al otro mundo... no puedes hacer nada, tu... Una persona mayor... yo no puedo hacer nada... se tiene que conformar con todo... Hay personas que no, hay personas que aunque sean mayores...échale guinda... pero bueno, hay de todo en la viña del señor...” (2d82c, 119:119).

Este estilo de vida no es característico de ninguna zona geográfica en concreto y conforma un grupo de población mayor que necesita de una atención profesional basada en un modelo de búsqueda ya que ellos no demandan y por lo tanto pueden pasar como los grandes olvidados, los invisibles.

\section{Conclusiones}

La perspectiva de la participación como una vía de fortalecimiento de la sociedad civil, aunque no está situada estrictamente en el campo gerontológico, permite comprender de qué forma las personas mayores participando cooperan en el desarrollo del capital social de nuestra sociedad, y cómo ello produce unos efectos en la construcción de valor público, de valor colectivo. Los beneficios que reporta a las personas mayores su incorporación en los procesos de participación, mejora su autovaloración, aumenta las formas de expresión de sus capacidades (pensar, expresarse, hacer...), de demostración de sus posibilidades de aprender y de aportar, así como incrementa el nivel de reconocimiento externo.

Para concluir nuestro trabajo queremos concretar propuestas de intervención que conduzcan a favorecer estilos de vida más activos y participativos en los diferentes contextos y que sirvan de ayuda a los profesionales responsables de los servicios de atención a las personas mayores.

1. Promover la participación social, potenciar la dinamización y acercar los instrumentos de participación a las personas que se mantienen más aisladas socialmente, principalmente, aquellas que viven la vejez como una etapa "natural" de la vida o como un "momento de abandono".

- mantener/reforzar/cuidar las redes de buena vecindad como instrumento social que entre otras cosas tiene la potencialidad de activar a las personas que se mantienen más aisladas, a través de las relaciones, la información, y el apoyo emocional.

- facilitar la movilidad de las personas para promocionar el acceso y la participación en actividades variadas aunque no se desarrollen en su municipio, sobretodo en las zonas de interior.

- dar apoyo/promover a las iniciativas asociativas con el objetivo de implicar y llegar a toda la población.

2. Potenciar el movimiento asociativo y espacios organizados de participación (centros sociales, cívicos), dar papel activo a personas mayores, especialmente, a aquellas personas mayores por su iniciativa corresponden con los perfiles de la vejez como una "oportunidad para el cambio" o la vejez como un "momento a compensar".

- facilitar el acceso a espacios y recursos para iniciar la actividad asociativa.

- otorgar un rol significativo en la comunidad e incrementar el reconocimiento social de las personas mayores.

- potenciar intercambios con personas de otros centros dentro y de fuera de Catalunya.

3. Orientar/facilitar actividades de ocio que promuevan estilos de vida saludables dirigidas a toda las personas mayores.

- promocionar la actividad física a través de actividades que resulten significativas a las personas mayores.

- favorecer relaciones interpersonales a través de la organización de actividades sociales y en grupo que resulten significativas

- organizar formación continuada: aprendizajes a lo largo de la vida para mantenerse actualizados en un mundo cambiante (nuevas tecnologías, idiomas, salud, dietas, etc.)

- dar asesoramiento en temas de que afectan a su vida cotidiana, entre otros, temas financieros, fiscales, legales, (voluntades anticipadas, herencias, testamento vital, incapacitaciones, tutelas, pensiones, etc.) 


\section{Referencias}

Baltes, P. B., Lindenberger, U. y Staudinger, U. M. (1998). Life-span theory in developmental psychology. En W. Damon (Ed.), R. M. Lerner, Handbook of child psychology 5 edition: Vol 1. Theoretical models of human development (pp. 1029-1143). Nueva York: Wiley.

Bauman, Z. (2005). Vida líquida. Barcelona, Paidós.

Berger, P. L. y Luckmann, T. (1968). La construcción social de la realidad. Buenos Aires, Amorrortu.

Bibeau, G. D., Pedersen, D. y Fuentes, G. (1985). Estilos de vida y sistemas de trabajo. Santiago de Chile: Editorial Universitaria.

Brandtstadter, I. y Lerner, R. M. (1999). Actions and self-development: Theory and research through the life span. Thousand Oaks: Sage.

Bronfenbrenner, U. (1987). La ecologia del desarrollo humano. Barcelona: Paidós.

Bruner, J. (1990). Actos de significado: más allá de la Revolución Cognitiva. Alianza Editorial: Madrid.

Burr, V. (1997). Introducció al construccionisme social. Barcelona: Proa.

Departament de Benestar Social (2002). Llibre blanc de la gent gran active: el paper actiu de la gent gran en la nova societat: 50 propostes de millora I 14 recomanacions. Barcelona: Generalitat de Catalunya.

Fidler, G. S. (1996). Life-style performance: from profile to conceptual model. The American Journal of Occupational Therapy, 50, 139-147.

García Sanz, B. (1996). La sociedad rural ante el siglo XXI. Madrid: Ministerio de Agricultura, Pesca y Alimentación.

García Sanz, B. (1997). Envejecimiento y mundo rural: problemas y soluciones. Madrid: IMSERSO.

Gergen, k. J. (1985). The social constructionist movement in modern psychology. American Psychologist 40, 266-275.

Golant, S. M. (2004). The urban-rural distinction in gerontology: An update of research. In H. W. Wahl, Scheidt R. \& Windley P. (Eds.), Aging in context:
Sociophysical environments. (pp. 280-312). Springer: Berlin, Heidelberg, New York.

Hink, S. (2004). The lived experience of oldest-old rural adults. Qualitative health research, 14, 779-791.

Horgas, A. L., Wilms, H. U. y Baltes, M. M. (1998). Daily life in very old age: everyday activities as expression of successful living. The Gerontologist, 38(5), 556-568.

Observatorio de Personas Mayores y Portal Mayores, (2009). Las personas mayores en España, Informe 2008. Madrid: IMSERSO.

Monreal, P. y Vilà, A. (2008). Programa Integral de Atención a las Personas Mayores en una zona rural. Anuario de Psicología. 39, 351-370.

Morse, Janice M. (1994). Designing Funded Qualitative Research, en Norman K. Denzin \& Yvonna S. Lincoln (Eds) Handbook of Qualitative Research. London: Sage, 220-235.

Neugarten, B. L. (1999). Los significados de la edad. Barcelona, Herder.

Observatorio de Personas Mayores (2004). Envejecimiento en el mundo rural: Necesidades singulares, políticas específicas. Boletín sobre el envejecimiento, 11 .

OMS, (2002). Envejecimiento activo: un marco político. Revista Española de Geriatría y Gerontología, 37(S2): 74-105.

Rodriguez, P. (1995). La investigación-acción-participativa (IAP) como estímulo de la participación de las personas mayores y del voluntariado social. Primera parte. En Rodríguez, P. y Colectivo IOE. Voluntariado y personas mayores. Una experiencia de IAP. Madrid: IMSERSO.

Smits, C. H., Deeg, D. M. y Schmand, B. (1999). Cognitive functioning and Health as determinants of mortality . American Journal of Epidemiology, 150, 978-986.

Taylor, S. \& Bogdan, R. (1986). Introducción a los métodos cualitativos de investigación. Barcelona: Paidós.

Whalley, L. y Deary, I. J. (2001). Longitudinal cohort study of childhood IQ and survival up to age 76 . British Medical Journal, 32.

Manuscrito recibido: 17/09/2009

Revisión recibida: 10/10/2009

Manuscrito aceptado: 20/10/2009 